\title{
Clinical utility of dronabinol in the treatment of weight loss associated with HIV and AIDS
}

This article was published in the following Dove Press journal:

HIVIAIDS - Research and Palliative Care

10 February 2016

Number of times this article has been viewed

\author{
Melissa E Badowski' \\ Sarah E Perez ${ }^{2}$ \\ 'Department of Pharmacy Practice, \\ Section of Infectious Diseases \\ Pharmacotherapy, College of \\ Pharmacy, University of Illinois at \\ Chicago, Chicago, IL, USA; ${ }^{2}$ Infectious \\ Diseases Clinic, Tufts Medical Center, \\ Boston, MA, USA
}

\begin{abstract}
Since the beginning of the HIV/AIDS epidemic, weight loss has been a common complaint for patients. The use of various definitions defining HIV wasting syndrome has made it difficult to determine its actual prevalence. Despite the use of highly active antiretroviral therapy, it is estimated that the prevalence of HIV wasting syndrome is between $14 \%$ and $38 \%$. HIV wasting syndrome may stem from conditions affecting chewing, swallowing, or gastrointestinal motility, neurologic disease affecting food intake or the perception of hunger or ability to eat, psychiatric illness, food insecurity generated from psychosocial or economic concerns, or anorexia due to medications, malabsorption, infections, or tumors. Treatment of HIV wasting syndrome may be managed with appetite stimulants (megestrol acetate or dronabinol), anabolic agents (testosterone, testosterone analogs, or recombinant human growth hormone), or, rarely, cytokine production modulators (thalidomide). The goal of this review is to provide an in-depth evaluation based on existing clinical trials on the clinical utility of dronabinol in the treatment of weight loss associated with HIV/AIDS. Although total body weight gain varies with dronabinol use ( -2.0 to $3.2 \mathrm{~kg}$ ), dronabinol is a well-tolerated option to promote appetite stimulation. Further studies are needed with standardized definitions of HIV-associated weight loss and clinical outcomes, robust sample sizes, safety and efficacy data on chronic use of dronabinol beyond 52 weeks, and associated virologic and immunologic outcomes.
\end{abstract}

Keywords: dronabinol, weight loss, HIV/AIDS, HIV wasting syndrome, cachexia

\section{Introduction}

Since the beginning of the HIV/AIDS epidemic, weight loss has been a common complaint for patients. The initial definition of HIV wasting syndrome was defined by the Centers for Disease Control and Prevention in 1987 as the involuntary weight loss of greater than $10 \%$ of baseline body weight in addition to 1) chronic weakness, 2) diarrhea (two or more loose stools per day), or 3 ) documented fever lasting for more than 1 month. ${ }^{1}$ Since then, updated definitions have become available to encompass rapid decreases in weight and designed to include low-weight individuals without available baseline weight that the previous definition would have excluded. In addition, the updated definition requires one of the following criteria with a diagnosis of HIV: 1) $10 \%$ unintentional weight loss over 12 months, 2) $7.5 \%$ unintentional weight loss over 6 months, or 3 ) unintentional weight loss of more than $5 \%$ over 3 months if other causes or factors of weight loss could not be ruled out. For individuals without documented baseline weight, those meeting the criteria for HIV wasting syndrome would include $5 \%$ body cell mass (BCM) loss within 6 months; in men: $\mathrm{BCM}<35 \%$ of total body weight (TBW) and body mass index (BMI) $<27 \mathrm{~kg} / \mathrm{m}^{2}$; in women: BCM
Correspondence: Melissa E Badowski Department of Pharmacy Practice, Section of Infectious Diseases Pharmacotherapy, College of Pharmacy, University of Illinois at Chicago, 833 SWood, M/C 164, Chicago, IL 606 I2, USA

Tel +I 3129966300

Fax + I 3123552823

Email badowski@uic.edu 
$<23 \%$ of TBW and BMI $<27 \mathrm{~kg} / \mathrm{m}^{2}$; or BMI $<20 \mathrm{~kg} / \mathrm{m}^{2}$ regardless of BCM in men and women. ${ }^{2}$ The most recent proposed definition of HIV wasting syndrome (TBW loss $>10 \%$ from initial visit, $\mathrm{BMI}<20 \mathrm{~kg} / \mathrm{m}^{2}$, or an unintentional TBW loss $>5 \%$ in 6 months and persisting for more than 1 year) focused on the rate of weight loss as well as the reality that patients present at different stages. ${ }^{3}$ Although the initial definition is no longer used, recent definitions have led to varying reports of incidence and prevalence of HIV wasting syndrome.

Prior to the availability of protease inhibitors, male AIDS patients weighed less than $90 \%$ of their ideal body weight or lost greater than $10 \%$ of their TBW. ${ }^{2}$ Since the development of highly active antiretroviral therapies (HAART), the incidence and prevalence of HIV wasting syndrome have declined but still remain a concern in patients living with HIV/AIDS. Despite successful initiation of HAART, the prevalence of weight loss and wasting was similar to those who were antiretroviral-naïve or failing HAART. ${ }^{4} \mathrm{HIV}$ wasting syndrome may stem from conditions affecting chewing, swallowing, or gastrointestinal motility, neurologic disease affecting food intake or the perception of hunger or ability to eat, psychiatric illness, food insecurity generated from psychosocial or economic concerns, or anorexia due to medications, malabsorption, infections, or tumors. ${ }^{5}$ The use of various definitions defining HIV wasting syndrome has made it difficult to determine its actual prevalence. Despite HAART, it is estimated that the prevalence of HIV wasting syndrome is between $14 \%$ and $38 \% .{ }^{3}$ Higher prevalence estimates for HIV wasting syndrome may be due to updated definitions compared to strict criteria that were initially proposed, which may have underestimated the true incidence and prevalence. In a managed care population receiving HAART, it was reported that between 2005 and 2007, the prevalence of HIV wasting syndrome was $8.3 \%$ and associated with significantly higher annual health-care costs as well as significantly more ambulatory care, and more emergency room and inpatient visits $(P<0.0001) .{ }^{6}$

When all definitions of HIV wasting syndrome were included, a significant risk of death was noted for each $1 \%$ increase in weight loss from baseline weight. Furthermore, an $11 \%$ increase in the risk of death was associated with each $1 \%$ increase in weight loss from the last visit. ${ }^{3}$ For patients not receiving HAART, a significant relationship was found for each $\log 10$ increase in HIV viral load that correlated with a $0.92 \mathrm{~kg}$ decrease in body weight $(P=0.003)$, yet weight change was not observed during HAART. This same study demonstrated that for every CD4 cell count decrease of 100 cells $/ \mathrm{mm}^{3}$, a $0.35 \mathrm{~kg}$ decrease in body weight was observed $(P<0.001) .^{7}$ Although there are no current treatment standards for the management of HIV wasting syndrome and weight loss, initiation of HAART and viral suppression tends to promote weight gain, on average, $10 \%-25 \%$ annually. ${ }^{8}$ For patients achieving virologic suppression but still experiencing weight loss despite HAART, pharmacologic intervention may be warranted to promote weight gain.

\section{Pathophysiology of HIV wasting syndrome}

The exact mechanism of how HIV causes weight loss is not well understood, yet causes may include inadequate caloric intake and changes in metabolism. Inadequate caloric intake may arise due to depression, financial hardships, inability to chew or swallow secondary to opportunistic infections, malabsorption (due to nausea or diarrhea), and medication toxicities. ${ }^{3,5}$ Changes in metabolism have been associated with HIV wasting syndrome and may stem from changes in metabolic demands, cytokine dysregulation, and hormonal changes. ${ }^{9}$ In patients with untreated HIV and subsequent weight loss, the resting energy expenditure was approximately $10 \%-35 \%$ greater (possibly higher in patients with opportunistic infections) compared with healthy individuals. ${ }^{10}$

Hypogonadism may contribute to weight loss in as many as $50 \%$ of males and females living with HIV. The mechanism associated with low testosterone levels was found to be associated with decreased protein synthesis, causing a decrease in lean body mass. Finally, for HIV-positive patients experiencing acute infections, cytokine (interleukin-6, interleukin-1b, and tumor necrosis factor- $\alpha$ ) production increased and was correlated with significant weight loss in HIV. ${ }^{3}$

\section{Treatments for HIV wasting syndrome}

Various treatment options exist for HIV wasting syndrome and include appetite stimulants (megestrol acetate, dronabinol, and mirtazapine), anabolic agents (testosterone, testosterone analogs, and recombinant human growth hormone $[\mathrm{rhGH}]$ ), and, rarely, cytokine production modulators (thalidomide). The most commonly used agents for initial management, upon virologic suppression, of HIV wasting syndrome are appetite stimulants since they promote weight gain and assist in minimizing anorexia. The decision of which agent(s) to choose should include comorbidities, drug-drug interactions, past medical history, and the ability to use and tolerate certain formulations. 


\section{Anabolic agents}

Anabolic agents such as testosterone, synthetic testosterone analogs, and rhGHs differ from appetite stimulants in that these agents increase muscle mass, which may account for weight gain. Testosterone and testosterone analogs are recommended in patients with primary and hypogonadotropic hypogonadism, repletion in instances of low levels, low libido, and HIV wasting (in the presence or absence of HAART). Testosterone and testosterone analogs increased TBW as much as $3.5 \mathrm{~kg} .{ }^{11}$ One benefit of testosterone and its analogs is the variety of available formulations. Testosterone levels should be monitored every 3-6 months after initiation of therapy and annually, thereafter. Although various metabolic, dermatologic, central nervous system (CNS), and endocrine adverse effects are associated with testosterone and testosterone analogs, minimal toxicities were reported in studies of HIV wasting syndrome.

rhGHs mimic natural human growth hormone in order to increase weight gain, since it minimizes the breakdown of protein and depletion of nitrogen. rhGHs should be reserved in severe cases since this requires a daily or every other day subcutaneous injection. Changes in TBW ranged between 0.7 and $3.2 \mathrm{~kg} .{ }^{12}$ Similar adverse effects seen with testosterone and testosterone analogs are associated with rhGHs and include arthralgia and myalgia.

\section{Cytokine production modulators}

The cytokine production modulator, thalidomide, is rarely used due to its significant toxicities and needs to be dispensed through a special restricted distribution program. Its effects on TBW gain has been reported to be $1.5-2.2 \mathrm{~kg}$ and is reserved for severe and refractory cases of HIV wasting syndrome due to its severe adverse effect profile (edema, thromboembolism, grade 3/4 rash, infection, liver function test abnormalities, diarrhea, hypersensitivity, fever, and peripheral neuropathy). ${ }^{13}$ Thalidomide is thought to exhibit its mechanism by acting as a TNF- $\alpha$ inhibitor by reducing TNF- $\alpha$ production and promoting TBW gain.

\section{Appetite stimulants} Megestrol acetate

Megestrol acetate is an oral suspension $(40 \mathrm{mg} / \mathrm{mL})$ or concentrated suspension $(125 \mathrm{mg} / \mathrm{mL})$ approved for the management of anorexia, cachexia, or unexplained weight loss associated with AIDS. Although the mechanism of action is unknown, megestrol acetate may enhance appetite stimulation by antagonizing the metabolic effects of catabolic cytokines. The recommended dose of megestrol acetate oral suspension is $400-800 \mathrm{mg} / \mathrm{d}$ (2-4 teaspoonfuls). Megestrol acetate ES (enhanced stability), also referred to as the concentrated suspension, has a recommended daily dose of 625 $\mathrm{mg}$ /day (1 teaspoonful). Megestrol acetate ES contains $0.06 \%$ ethanol and is associated with rapid dissolution and increased absorption when compared to the oral suspension (40 mg/ $\mathrm{mL}$ ). In addition, this formulation requires a smaller volume of liquid, which is beneficial for those with dysphagia or anorexia. Both suspension formulations can be stored at room temperature (between $15^{\circ} \mathrm{C}$ and $25^{\circ} \mathrm{C}$ ). On average, megestrol acetate has been associated with an increase of 4-5 kg (range: $0.863-7.3 \mathrm{~kg}$ ) in TBW over a period of 12 weeks. ${ }^{12}$

Although rarely used, the protease inhibitor, indinavir, experienced a significant decrease in exposure when combined with megestrol acetate. It is recommended to increase the dose of indinavir if combination therapy with megestrol acetate is used. Megestrol acetate has also been found to reduce anticoagulant effects. Therefore, careful consideration should occur prior to initiation of megestrol acetate, because of the increased risk of procoagulant effects and thromboembolism. Megestrol acetate is contraindicated with the use of dofetilide since it may increase the serum concentration of dofetilide.

Reported adverse effects with megestrol acetate use include thromboembolism, adrenal insufficiency, and metabolic disorders. In patients receiving protease inhibitors, there may be an increased risk for overlapping toxicities such as adrenal insufficiency and hyperglycemia.

\section{Mirtazapine}

Although not approved for HIV wasting syndrome, mirtazapine is a tetracyclic antidepressant that is available as an oral tablet or an orally disintegrating tablet. Despite weight gain being reported as a common adverse effect of mirtazapine, it is for this reason that mirtazapine may be considered in HIV wasting syndrome in patients with concomitant depression. One study evaluated the use of mirtazapine in HIV-positive patients and found a $3 \mathrm{~kg}$ increase in TBW over a period of 12 weeks. ${ }^{14}$ Although the sample size was relatively small and duration of study was short, it demonstrated that mirtazapine may be another treatment option for managing weight loss in HIV patients with depression. Appetite stimulation associated with mirtazapine use is thought to be secondary to blocking serotonin 5-HT1b and 5-HT2 receptors as well as inhibiting the release of neuropeptide Y. ${ }^{15}$ Mirtazapine has also been associated with antiemetic effects through inhibition of 5-HT3 receptors. 
It is recommended to initiate mirtazapine at $15 \mathrm{mg}$ daily and titrate to effect. The daily dose of mirtazapine should not exceed $60 \mathrm{mg}$ daily. Titration to therapeutic effect should occur every 1-2 weeks based on safety and tolerability.

If coadministered with efavirenz, mirtazapine may increase the likelihood of toxicities and CNS adverse effects such as drowsiness. Since mirtazapine is a substrate of CYP2D6, CYP1A2, and CYP3A4, coadministration with efavirenz may lead to inhibitory or inducing effects of mirtazapine, whereas protease inhibitors may lead to inhibitory effects of mirtazapine. ${ }^{15}$ In the clinical setting, these agents are frequently administered concomitantly but agents should be monitored for the increased development of adverse effects. Other agents that act as CNS depressants should be administered cautiously with mirtazapine because of its enhanced CNS depressant effects. Additional adverse effects associated with mirtazapine use may include increased serum cholesterol and triglyceride levels, constipation, xerostomia, and orthostatic hypotension.

\section{Dronabinol (an in-depth review)}

Dronabinol is an orally active cannabinoid that acts on the CNS. Dronabinol is a synthetic form of delta-9-tetrahydrocannabinol (THC), which is a naturally occurring component of Cannabis sativa $L$ and activated cannabinoid receptors (CB) $\mathrm{CB} 1$ and $\mathrm{CB} 2$. Although dronabinol has a similar affinity for $\mathrm{CB} 1$ and $\mathrm{CB} 2$ receptors, efficacy is less at $\mathrm{CB} 2$ receptors. When $\mathrm{CB} 1$ is activated, marijuana-like effects occur and provide appetite enhancement, analgesia, and muscle relaxation. ${ }^{16}$ The same effects are not seen when CB2 activation occurs. Dronabinol acts on CB1 receptors in the CNS to produce affective, sensory, somatic, and cognitive psychological effects as well as central sympathomimetic activity manifesting as tachycardia and/or conjunctival injection. Occasional reports of orthostatic hypotension and/or syncopal episodes due to dronabinol were described, but these subsided within 12 days of treatment initiation. ${ }^{17}$ Dronabinol appears to have reversible effects on appetite, mood, cognition, memory, and perception and occur with higher frequency in patients on higher doses.

After administration, the onset of action is approximately 0.5-1 hour and peak effect is 2-4 hours after administration. Psychoactive effects last 4-6 hours, while the appetite stimulant effect of dronabinol may continue for 24 hours or longer after administration. ${ }^{17}$ Although tachyphylaxis and tolerance may develop to some pharmacologic effects of dronabinol and other cannabinoids with chronic use, they are not expected to develop to the appetite stimulant effect of dronabinol. The most common adverse effects attributed to dronabinol are dose-dependent CNS effects, including abnormal thinking, dizziness, somnolence, and paranoia. Caution should be used when operating machinery or driving with dronabinol use as it may impair physical or mental abilities. Caution should also be observed in patients with a history of substance abuse because of the potential development of tolerance and dependence with prolonged use of dronabinol, but as previously mentioned it is unlikely to occur to the appetite stimulant properties of dronabinol. For patients with a history of seizure disorder, the development of new seizure activity should prompt discontinuation of dronabinol as it has the potential to lower seizure threshold.

Dronabinol is available as $2.5,5$, and $10 \mathrm{mg}$ oil-filled, oral soft gelatin capsules. Dronabinol must be stored in a cool or refrigerated environment (between $8^{\circ} \mathrm{C}$ and $15^{\circ} \mathrm{C}$ ). When used for its appetite stimulant properties in the management of weight loss in HIV/AIDS, the initial dose of dronabinol is $2.5 \mathrm{mg}$ by mouth twice daily before lunch and dinner. In patients unable to tolerate the initial dose, dronabinol may be reduced to $2.5 \mathrm{mg}$ by mouth once daily in the evening or at bedtime. The dose of dronabinol may be titrated gradually to response and tolerability to a maximum daily dose of 10 mg by mouth twice daily. The use of dronabinol should be avoided in individuals with a history of hypersensitivity to cannabinoids or sesame oil.

Oral absorption of dronabinol is between $90 \%$ and $95 \%$, of which $10 \%-20 \%$ reaches systemic circulation. Since it is highly lipid soluble, is has a rather large volume of distribution, which is approximately $10 \mathrm{~L} / \mathrm{kg}$. Dronabinol and its metabolites are estimated to be $97 \%$ protein bound. Dronabinol's elimination phase uses a two-compartment model where the initial half-life is approximately 4 hours and the terminal half-life is $25-36$ hours. Due to the large volume of distribution, dronabinol and its metabolites may be excreted at low levels for prolonged periods of time. ${ }^{17}$

Although major drug interactions do not exist with dronabinol, dronabinol acts as a minor substrate for CYP2C9 and CYP3A4. Therefore, therapy with concomitant agents should be monitored for increased signs and symptoms of toxicity (CNS depressant effects, tachycardia, orthostasis) correlating with increased serum concentrations of dronabinol.

\section{Efficacy and safety data for dronabinol}

Various clinical evaluations have been conducted with the use of dronabinol (alone or in combination with other agents) and its effects on TBW gain. To date, 10 studies have evaluated 
the use of dronabinol for the management of HIV wasting syndrome. Most of these evaluations lack standard definitions for HIV wasting syndrome, inclusion/exclusion criteria, study design, ART, and reporting of clinical outcomes. Furthermore, many of these studies do not include current ART with respect to current practices, sample sizes robust enough to detect significant differences, as well as long-term safety and efficacy data with dronabinol. An extensive review of each study can be found in Table $1 .^{18-27}$

Although safety and efficacy data have been documented, change in TBW with the use of dronabinol only ranged from -2.0 to $3.2 \mathrm{~kg}$ (Table 1). ${ }^{18-23,25,26}$ In studies with placebo controls, subjects generally lost weight when receiving placebo (range: -0.7 to $1.1 \mathrm{~kg}$ ) (Table 1). ${ }^{19,20,23}$ One study evaluated the use of dronabinol and megestrol acetate alone or in combination and found a statistically significant increase in TBW for megestrol acetate $750 \mathrm{mg}$ daily and megestrol acetate $750 \mathrm{mg}$ daily plus dronabinol $2.5 \mathrm{mg}$ twice daily $(P<0.0001)$ but did not find any significant difference in TBW with dronabinol $2.5 \mathrm{mg}$ twice daily or dronabinol $2.5 \mathrm{mg}$ twice daily plus megestrol acetate $250 \mathrm{mg}$ daily (Table 1). ${ }^{21}$ Inhaled marijuana, in the form of a cigarette, was evaluated alone or in combination with dronabinol but no statistically significant changes in TBW in any treatment group was found. Haney et $\mathrm{a}^{24,25}$ evaluated the effects of dronabinol and marijuana in HIV-positive marijuana smokers and concluded that both dronabinol (4-8 times the recommended dose) and marijuana were well tolerated and produced substantial increases in food intake.

Dronabinol has been well tolerated at doses up to $20 \mathrm{mg} / \mathrm{d}$ and had mild-to-moderate CNS effects such as dizziness, euphoria, and sedation. ${ }^{18-20,22,24-27}$ One study demonstrated a clinically significant difference in the number of subjects reporting anxiety/nervousness with dronabinol $2.5 \mathrm{mg}$ twice daily $(\mathrm{n}=25)$ compared to placebo $(\mathrm{n}=9)(P<0.001),{ }^{20}$ while another study demonstrated a statistically significant increase in heart rate in subjects receiving $\geq 20 \mathrm{mg} /$ day of dronabinol (20 mg, $P<0.01 ; 30 \mathrm{mg}, P<0.0001$ ) (Table 1). ${ }^{24}$ It is interesting to note that this effect occurred only in subjects with a normal bioelectrical impedance analysis and measurement of body fat in relation to lean body mass.

\section{Quality of life}

Various quality-of-life measures have been improved in patients treated with dronabinol. When compared to placebo, appetite increased from baseline by $37 \%$ in those receiving dronabinol compared to only $17 \%$ with placebo $(P=0.05) .{ }^{20}$ In the same study, for patients who completed at least 4 weeks of treatment and took $75 \%$ of their study medication, appetite increased from $38 \%$ compared to $8 \%$ receiving placebo $(P=0.015)$. Improvement in mood was observed in $10 \%$ of patients on dronabinol compared to $2 \%$ decline in patients receiving placebo $(P=0.06)$, and nausea decreased in $22 \%$ of those receiving dronabinol compared to $4 \%$ in the placebo $\operatorname{arm}(P=0.26)$. Patients were also evaluated for Karnofsky performance status to determine patients' general well-being and activities of daily life. On a scale from 0 (representing death) to 100 (indicating normal activity), patients receiving dronabinol had a drop in Karnofsky scores by 2.5 points compared to no change in those receiving placebo $(P=0.18) .{ }^{20}$

Another study evaluated quality of life by comparing four arms: 1) dronabinol $2.5 \mathrm{mg}$ BID, 2) megestrol acetate $750 \mathrm{mg}$ daily, 3) megestrol acetate $750 \mathrm{mg}$ daily + dronabinol $2.5 \mathrm{mg} \mathrm{BID}, 4)$ megestrol acetate $250 \mathrm{mg}$ daily + dronabinol $2.5 \mathrm{mg} \mathrm{BID} .^{21}$ No significant changes over time were identified nor any difference between the treatment arms in terms of Karnofsky performance status, functional assessment of HIV infection score, visual analog scale for mood, or visual analog scale for nausea. Among the entire study population, the visual analog scale (VAS) for hunger improved after the first week of therapy, with a weekly sum score for breakfast decreasing from 170 to $130(P=0.0002)$, for lunch from 183 to 112 ( $P=0.0001)$, and for dinner from 192 to $114(P=0.0001)$. There were no differences between groups and no significant change after the first week. For perception scores on a scale of 0 (no improvement) to 10 (improvement), improvement in social and family life increased from 6.8 at baseline to 7.8 at week 4 between all study groups $(P=0.005)$ and improvements in additional unspecified concerns affecting quality of life from 7 at baseline to 8 after 4 weeks $(P=0.003)$.

In a study evaluating short-term, high-dose dronabinol (10 mg four times daily) plus inhaled marijuana compared to placebo plus inhaled marijuana, dronabinol significantly increased VAS positive effect on days 1-8 and days 9-16 $(P<0.01)$ as well as sleep efficiency on days $1-8(P<0.01) .{ }^{27}$ No significant effect was seen on VAS anxiety, somatic symptoms, or negative effects. Although dronabinol has been associated with improved quality of mood and sleep, additional studies are needed to evaluate prolonged use of dronabinol on these parameters.

There is limited data evaluating patient satisfaction and acceptability of dronabinol use. One study compared dronabinol and marijuana use in various doses for HIV-positive marijuana smokers. ${ }^{25}$ Ratings of "good drug effect," "mellow," and "high" were significantly increased after taking dronabinol $10 \mathrm{mg}$ compared to dronabinol $5 \mathrm{mg}$ in those also 


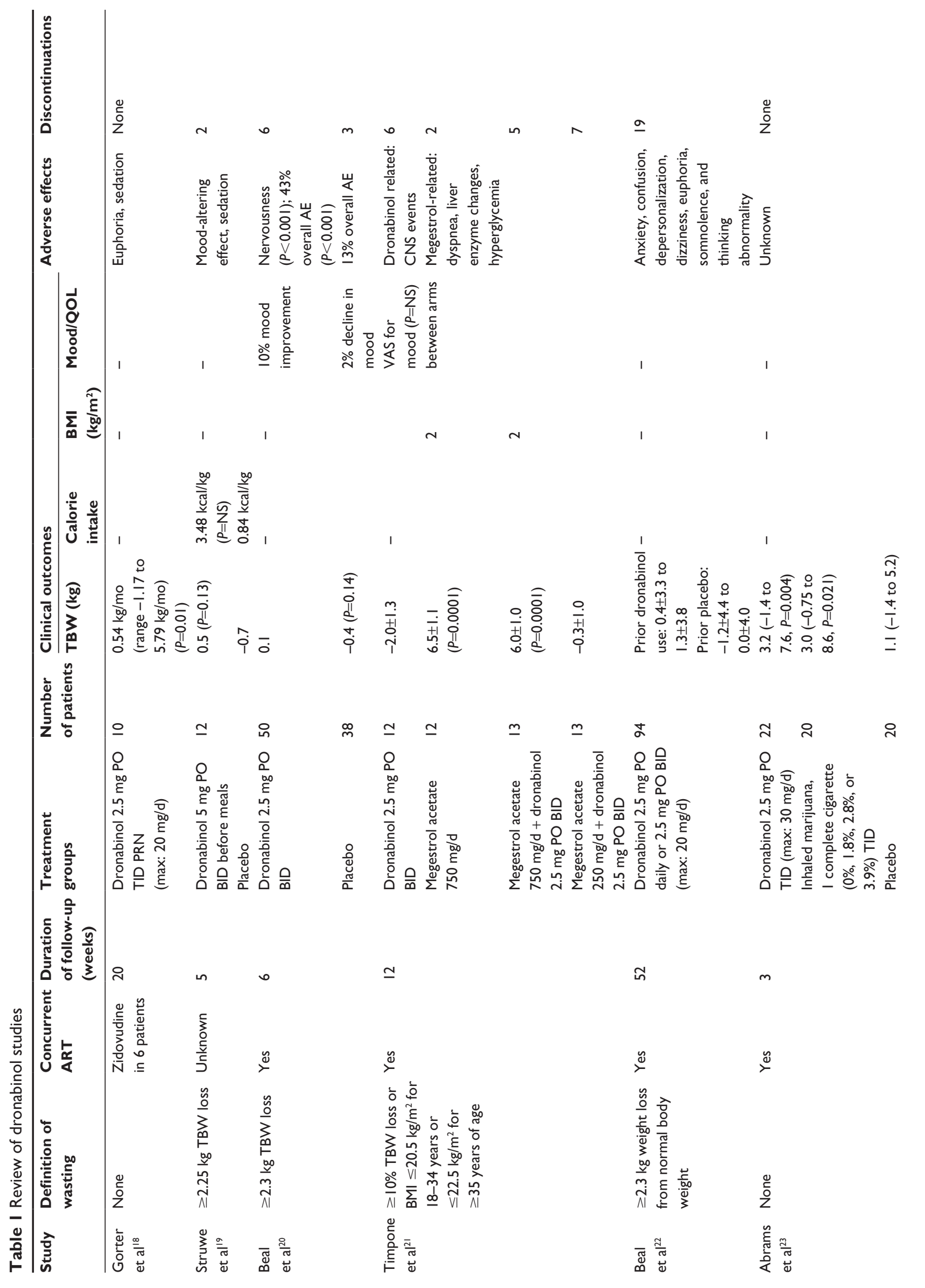


$\stackrel{̊}{\mathrm{c}}$

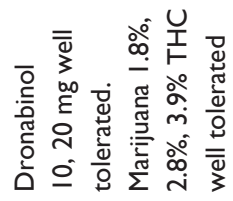

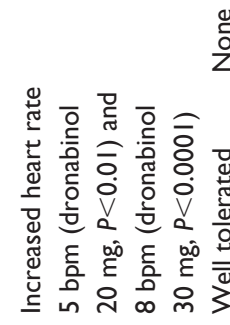

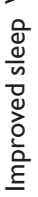

$\begin{array}{ll}\frac{5}{3} & \frac{5}{5} \\ \frac{5}{5} & \frac{5}{5} \\ 5 & \end{array}$

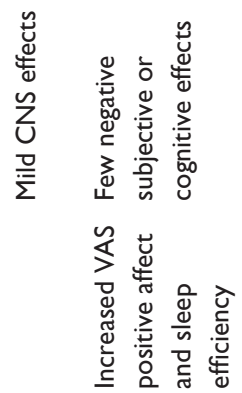

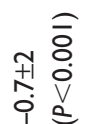

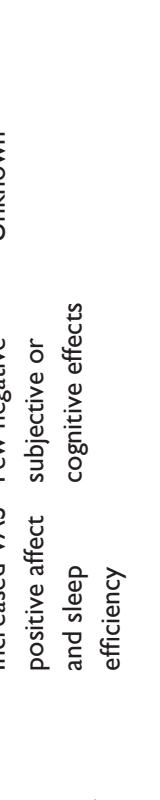

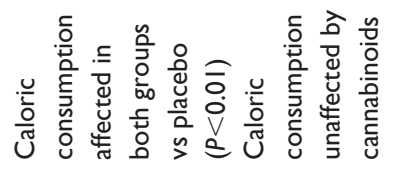

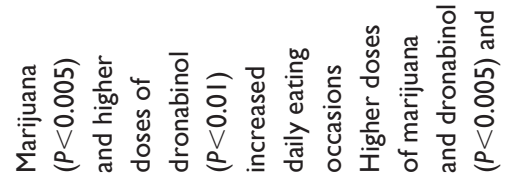

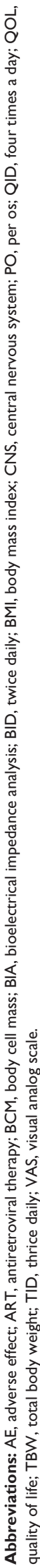

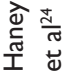

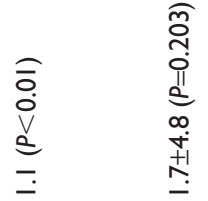

$\stackrel{+1}{\stackrel{+}{*}}$

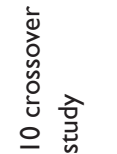

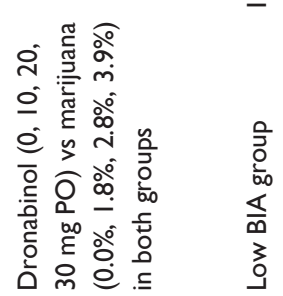

$\underline{n}$

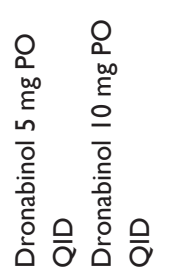

$\circ$

$\stackrel{\check{s}}{\nu}$

$\stackrel{0}{\check{0}}$

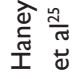

$\stackrel{0}{\check{0}} \stackrel{0}{\check{0}}$

离苟苟苟

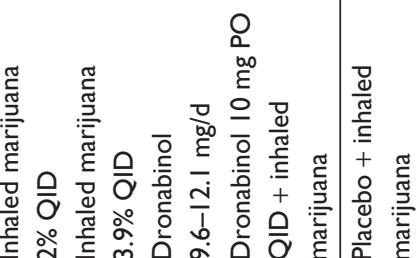

$\stackrel{y}{\check{y}}$

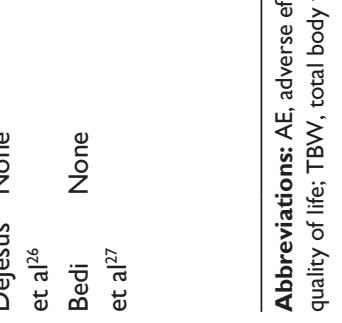


receiving marijuana doses of $2 \%$ or $3.9 \%$ THC $(P<0.005)$. Those taking the dronabinol $10 \mathrm{mg}$ dose and $3.9 \%$ THC marijuana had increased dronabinol ratings for good effect, liking, strength, and willingness to take dronabinol again. Dronabinol $10 \mathrm{mg}$ did have significant increased ratings of inability to concentrate $(P<0.01)$. More studies are needed that evaluate acceptability and subjective effects of dronabinol use.

\section{Conclusion}

Although the incidence of HIV wasting has decreased since the introduction of HAART, those who continue to be affected by HIV wasting syndrome require treatment to limit associated morbidity and mortality. In individuals with HIV/ AIDS who have decreased appetite and weight loss unattributed to other comorbidities, such as androgen deficiency or depression, dronabinol is a well-tolerated option to promote appetite stimulation. Although TBW gain varies with dronabinol use ( -2.0 to $3.2 \mathrm{~kg}$ ), a benefit of dronabinol use allows for standardized THC levels and is not associated with a rapid high unlike smoked or inhaled marijuana. Many of the adverse effects seen with dronabinol are dose dependent. Dosing of patients should be individualized based on appetite stimulation and tolerability. Limitations for dronabinol use include individuals unable to take an oral capsule, unable to maintain dronabinol in a cool or refrigerated environment, or those with hypersensitivity to any components of dronabinol (including sesame oil). Further studies are needed with standardized definitions of HIV-associated weight loss and clinical outcomes, robust sample sizes, safety and efficacy data on chronic use of dronabinol beyond 52 weeks, and associated virologic and immunologic outcomes.

\section{Disclosure}

The authors report no conflicts of interest in this work.

\section{References}

1. Centers for Disease Control. Revision of the CDC surveillance case definition for acquired immunodeficiency syndrome. Council of State and Territorial Epidemiologists; AIDS Program, Center for Infectious Diseases. MMWR Morb Mortal Wkly Rep. 1987;36(Suppl 1):S1-S15.

2. Polsky B, Kotler D, Steinhart C. HIV-associated wasting in the HAART era: guidelines for assessment, diagnosis, and treatment. AIDS Patient Care STDS. 2001;15:411-423.

3. Mangili A, Murman DH, Zampini AM, Wanke CA. Nutrition and HIV infection: review of weight loss and wasting in the era of highly active antiretroviral therapy from the nutrition for health living cohort. Clin Infect Dis. 2006;42:836-842.

4. Tang AM, Jacobson DL, Spiegelman D, Knox TA, Wanke CA. Increasing risk of $5 \%$ or greater unintentional weight loss in a cohort of HIV-infected patients, 1995 to 2003. J Acquir Immune Defic Syndr. 2005;40:70-76.
5. Mankal PK, Kotler DP. From wasting to obesity, changes in nutritional concerns in HIV/AIDS. Endocrinol Metab Clin North Am. 2014;43:647-663.

6. Siddiqui J, Phillips AL, Freedland ES, Sklar AR, Darkow T, Harley CR. Prevalence and cost of HIV-associated weight loss in a managed care population. Curr Med Res Opin. 2009;25(5):1307-1317.

7. Mwamburi DM, Wilson IB, Jacobson DL, et al. Understanding the role of HIV load in determining weight change in the era of highly active antiretroviral therapy. Clin Infect Dis. 2005;40(1):167-173.

8. AIDS InfoNet. Wasting Syndrome. Available from: http://www.aidsinfonet.org/fact_sheets/view/519pml.htm. Accessed November 22, 2015.

9. Grinspoon S, Mulligan K. Weight loss and wasting in patients infected with human immunodeficiency virus. Clin Infect Dis. 2003;36(Suppl 2): S69-S78.

10. Kosmiski L. Energy expenditure in HIV infection. Am J Clin Nutr. 2001;94(6):1677S-1682S.

11. Hengge UR, Stocks K, Wiehler H, et al. Double-blind, randomized, placebo-controlled phase III trial of oxymetholone for the treatment of HIV wasting. AIDS. 2003;17:699-710.

12. Badowski M, Pandit N. Pharmacologic management of human immunodeficiency virus wasting syndrome. Pharmacotherapy. 2014;34(8):868-861.

13. Kaplan G, Thomas S, Fierer DS, et al. Thalidomide for the treatment of AIDS-associated wasting. AIDS Res Hum Retroviruses. 2000;16:1345-1355.

14. Elliott AJ, Roy-Byrne PP. Mirtazapine for depression in patients with human immunodeficiency virus. J Clin Psychopharmacol. 2000;20:265-267.

15. Remeron (mirtazapine) [package insert]. White Station, NJ: Merck and Co., Inc.; 2011.

16. Grotenhermen F. Pharmacokinetics and pharmacodynamics of cannabinoids. Clin Pharmacokinet. 2003;42(2):327-360.

17. Marinol (dronabinol) [package insert]. North Chicago, IL: AbbVie, Inc.; 2013.

18. Gorter R, Seefried M, Volberding P. Dronabinol effects on weight in patients with HIV infection. AIDS. 1992;6(1):127.

19. Struwe M, Kaempfer SH, Geiger CJ, et al. Effect of dronabinol on nutritional status in HIV infection. Ann Pharmacother. 1993;27(7-8):827-831.

20. Beal JE, Olson R, Laubenstein L, et al. Dronabinol as a treatment for anorexia associated with weight loss in patients with AIDS. J Pain Symptom Manage. 1995;10(2):89-97.

21. Timpone JG, Wright DJ, Li N, et al. The safety and pharmacokinetics of single-agent and combination therapy with megestrol acetate and dronabinol for the treatment of HIV wasting syndrome. AIDS Res Hum Retroviruses. 1997;13(4):305-315.

22. Beal JE, Olson R, Lefkowitz L, et al. Long-term efficacy and safety of dronabinol for Acquired Immunodeficiency Syndrome-associated anorexia. J Pain Symptom Manage. 1997;14(1):7-14.

23. Abrams DI, Hilton JF, Leiser RJ. Short-term effects of cannabinoids in patients with HIV-1 infection. Ann Intern Med. 2003;139: 258-266.

24. Haney M, Rabkin J, Gunderson E, Foltin RW. Dronabinol and marijuana in HIV+ marijuana smokers: acute effects on caloric intake and mood. Psychopharmacology (Berl). 2005;181:170-178.

25. Haney M, Gunderson EW, Rabkin J, et al. Dronabinol and marijuana in HIV-positive marijuana smokers: caloric intake, mood, and sleep. J Acquir Immune Defic Syndr. 2007;45(5):545-554.

26. DeJesus E, Rodwick BM, Bowers D, Cohen CJ, Pearce D. Use of dronabinol improves appetite and reverses weight loss in HIV/AIDSinfected patients. J Int Assoc Physicians AIDS Care. 2007;6(2): 95-100.

27. Bedi G, Foltin RW, Gunderson EW, et al. Efficacy and tolerability of high-dose dronabinol maintenance in HIV-positive marijuana smokers: a controlled laboratory study. Psychopharmacology (Berl). 2010;212(4):675-686. 
HIV/AIDS - Research and Palliative Care

Dovepress

\section{Publish your work in this journal}

HIV/AIDS - Research and Palliative Care is an international, peerreviewed open-access journal focusing on advances in research in HIV, its clinical progression and management options including antivira treatment, palliative care and public healthcare policies to control viral spread. The journal welcomes original research, basic science, clinical \& epidemiological studies, reviews \& evaluations, expert opinion \& commentary, case reports \& extended reports. The manuscript management system is completely online and includes a very quick and fair peer-review system. Visit http://www.dovepress.com/ testimonials.php to read real quotes from published authors.

Submit your manuscript here: http://www.dovepress.com/hivaids---research-and-palliative-care-journal 